\title{
Zhedanov's Algebra $A W(3)$ and the Double Affine Hecke Algebra in the Rank One Case. II. The Spherical Subalgebra
}

Tom H. KOORNWINDER

Korteweg-de Vries Institute, University of Amsterdam, Plantage Muidergracht 24, 1018 TV Amsterdam, The Netherlands

E-mail: thk@science.uva.nl

URL: http://www.science.uva.nl/ thk/

Received November 15, 2007, in final form June 03, 2008; Published online June 10, 2008

Original article is available at http://www.emis.de/journals/SIGMA/2008/052/

\begin{abstract}
This paper builds on the previous paper by the author, where a relationship between Zhedanov's algebra $A W(3)$ and the double affine Hecke algebra (DAHA) corresponding to the Askey-Wilson polynomials was established. It is shown here that the spherical subalgebra of this DAHA is isomorphic to $A W(3)$ with an additional relation that the Casimir operator equals an explicit constant. A similar result with $q$-shifted parameters holds for the antispherical subalgebra. Some theorems on centralizers and centers for the algebras under consideration will finally be proved as corollaries of the characterization of the spherical and antispherical subalgebra.
\end{abstract}

Key words: Zhedanov's algebra $A W(3)$; double affine Hecke algebra in rank one; AskeyWilson polynomials; spherical subalgebra

2000 Mathematics Subject Classification: 33D80

\section{Introduction}

Zhedanov [15] introduced in 1991 an algebra $A W(3)$ with three generators $K_{0}, K_{1}, K_{2}$ and three relations in the form of $q$-commutators, which describes deeper symmetries of the Askey-Wilson polynomials. In the basic representation (or polynomial representation) of $A W(3)$ on the space of symmetric Laurent polynomials in $z, K_{0}$ acts as the second order $q$-difference operator $D_{\text {sym }}$ for which the Askey-Wilson polynomials are eigenfunctions and $K_{1}$ acts as multiplication by $z+z^{-1}$. The Casimir operator $Q$ for $A W(3)$ becomes a scalar $Q_{0}$ in this representation. Let $A W\left(3, Q_{0}\right)$ be $A W(3)$ with the additional relation $Q=Q_{0}$. Then the basic representation $A W\left(3, Q_{0}\right)$ is faithful, see [7]. There is a parameter changing anti-algebra isomorphism of $A W(3)$ which interchanges $K_{0}$ and $K_{1}$, and hence interchanges $D_{\text {sym }}$ and $z+z^{-1}$ in the basic representation. In the basic representation this duality isomorphism can be realized by an integral transform having the Askey-Wilson polynomial $P_{n}[z]$ as kernel which maps symmetric Laurent polynomials to infinite sequences $\left\{c_{n}\right\}_{n=0,1, \ldots}$.

In 1992 Cherednik [2] introduced double affine Hecke algebras associated with root systems (DAHA's). This was the first of an important series of papers by the same author, where a representation of the DAHA was given in terms of $q$-difference-reflection operators ( $q$-analogues of Dunkl operators), joint eigenfunctions of such operators were identified as non-symmetric Macdonald polynomials, and Macdonald's conjectures for ordinary (symmetric) Macdonald polynomials associated with root systems could be proved. The idea of nonsymmetric polynomials

\footnotetext{
${ }^{\star}$ This paper is a contribution to the Proceedings of the Seventh International Conference "Symmetry in Nonlinear Mathematical Physics" (June 24-30, 2007, Kyiv, Ukraine). The full collection is available at http://www.emis.de/journals/SIGMA/symmetry2007.html
} 
was very fruitful, on the one hand as an important extension of traditional harmonic analysis involving orthogonal systems of special functions, on the other hand because the nonsymmetric point of view was helpful for understanding the symmetric case better. For instance, a duality anti-algebra isomorphism and shift operators (as studied earlier by Opdam [11] in the symmetric framework) occur naturally in the non-symmetric context.

Related to Askey-Wilson polynomials the DAHA of type $\left(C_{1}^{\vee}, C_{1}\right)$ (four parameters) was studied by Sahi [13, 14], Noumi \& Stokman [9], and Macdonald [8, Ch. 6]. See also the author's previous paper [7]. The same phenomena as described in the previous paragraph occur here, but in a very explicit form. See for instance Remarks 2.7 and 4.5 (referring to [9]) about the duality anti-algebra isomorphism and the shift operators, respectively.

In [7] I also discussed how the algebra $A W\left(3, Q_{0}\right)$ is related to the double affine Hecke algebra (DAHA) of type $\left(C_{1}^{\vee}, C_{1}\right)$. In the basic (or polynomial) representation of this DAHA (denoted by $\tilde{\mathfrak{H}})$ on the space of Laurent polynomials in $z$, the nonsymmetric Askey-Wilson polynomials occur as eigenfunctions of a suitable element $Y$ of $\tilde{\mathfrak{H}}$ (see [13, 9], [8, Ch. 6]). It turns out (see [7]) that a central extension $\widetilde{A W}\left(3, Q_{0}\right)$ of $A W\left(3, Q_{0}\right)$ can be embedded as a subalgebra of $\tilde{\mathfrak{H}}$. As pointed out in the present paper (see Remark 2.7), the duality anti-algebra isomorphisms for $\widetilde{A W}\left(3, Q_{0}\right)$ and $\tilde{\mathfrak{H}}$ are compatible with this embedding.

It would be interesting, also for possible generalisations to higher rank, to have a more conceptual way of decribing the relationship between Zhedanov's algebra and the double affine Hecke algebra. The present paper establishes this by showing that the algebra $A W\left(3, Q_{0}\right)$ is isomorphic to the spherical subalgebra of $\tilde{\mathfrak{H}}$. The definition of a spherical subalgebra of a DAHA goes back to Etingof \& Ginzburg [3], where a similar object was defined in the context of Cherednik algebras, see also [1]. The definition of spherical subalgebra for the DAHA of type $\left(C_{1}^{\vee}, C_{1}\right)$ was given by Oblomkov [10]. In general, the spherical subalgebra of a DAHA $\tilde{\mathfrak{H}}$ is the algebra $P_{\text {sym }} \tilde{\mathfrak{H}} P_{\text {sym }}$, where $P_{\text {sym }}$ is the symmetrizer idempotent in $\tilde{\mathfrak{H}}$.

The proof of the isomorphism of $A W\left(3, Q_{0}\right)$ with $P_{\text {sym }} \tilde{\mathfrak{H}} P_{\text {sym }}$ in Section 3 is somewhat technical. It heavily uses the explicit relations given in [7] for the algebras $A W\left(3, Q_{0}\right), \widetilde{A W}\left(3, Q_{0}\right)$ and $\tilde{\mathfrak{H}}$. In Section 4 a similar isomorphism is proved between $A W\left(3, Q_{0}\right)$ with two of its parameters $q$-shifted and $P_{\text {sym }}^{-} \tilde{\mathfrak{H}} P_{\text {sym }}^{-}$, where $P_{\text {sym }}^{-}$is the antisymmetrizing idempotent in $\tilde{\mathfrak{H}}$. In the final Section 5 it is shown as a corollary of these two isomorphisms that $\widetilde{A W}\left(3, Q_{0}\right)$ is isomorphic with the centralizer of $T_{1}$ in $\tilde{\mathfrak{H}}$. There it is also shown that the center of $A W\left(3, Q_{0}\right)$ is trivial, with a proof in the same spirit as the proof of the faithfulness of the basic representation of $A W\left(3, Q_{0}\right)$ given in [7]. Combination of the various results finally gives as a corollary that the center of $\tilde{\mathfrak{H}}$ is trivial.

\section{Conventions}

Throughout assume that $q$ and $a, b, c, d$ are complex constants such that

$$
q \neq 0, \quad q^{m} \neq 1(m=1,2, \ldots), \quad a, b, c, d \neq 0, \quad a b c d \neq q^{-m}(m=0,1,2, \ldots) .
$$

Let $e_{1}, e_{2}, e_{3}, e_{4}$ be the elementary symmetric polynomials in $a, b, c, d$ :

$$
\begin{aligned}
& e_{1}:=a+b+c+d, \quad e_{2}:=a b+a c+b c+a d+b d+c d, \\
& e_{3}:=a b c+a b d+a c d+b c d, \quad e_{4}:=a b c d .
\end{aligned}
$$

For Laurent polynomials $f$ in $z$ the $z$-dependence will be written as $f[z]$. Symmetric Laurent polynomials $f[z]=\sum_{k=-n}^{n} c_{k} z^{k}$ (where $c_{k}=c_{-k}$ ) are related to ordinary polynomials $f(x)$ in $x=\frac{1}{2}\left(z+z^{-1}\right)$ by $f\left(\frac{1}{2}\left(z+z^{-1}\right)\right)=f[z]$. 


\section{Summary of earlier results}

This section summarizes some results from [7], while Remarks 2.1 and 2.7 about the duality anti-algebra isomorphism are new here.

\subsection{Askey-Wilson polynomials}

The Askey-Wilson polynomials are given by

$$
p_{n}\left(\frac{1}{2}\left(z+z^{-1}\right) ; a, b, c, d \mid q\right):=\frac{(a b, a c, a d ; q)_{n}}{a^{n}}{ }_{4} \phi_{3}\left(\begin{array}{c}
q^{-n}, q^{n-1} a b c d, a z, a z^{-1} \\
a b, a c, a d
\end{array} ; q, q\right)
$$

(see [4] for the definition of $q$-shifted factorials $(a ; q)_{n}$ and of $q$-hypergeometric series $\left.{ }_{r} \phi_{s}\right)$. These polynomials are symmetric in $a, b, c, d$. We will work with the renormalized version which is monic as a Laurent polynomial in $z$ (i.e., the coefficient of $z^{n}$ equals 1 ):

$$
P_{n}[z]=P_{n}[z ; a, b, c, d \mid q]:=\frac{1}{\left(a b c d q^{n-1} ; q\right)_{n}} p_{n}\left(\frac{1}{2}\left(z+z^{-1}\right) ; a, b, c, d \mid q\right) .
$$

The polynomials $P_{n}[z]$ are eigenfunctions of the operator $D_{\text {sym }}$ acting on the space $\mathcal{A}_{\text {sym }}$ of symmetric Laurent polynomials $f[z]=f\left[z^{-1}\right]$ :

$$
\begin{aligned}
\left(D_{\text {sym }} f\right)[z]:= & \frac{(1-a z)(1-b z)(1-c z)(1-d z)}{\left(1-z^{2}\right)\left(1-q z^{2}\right)}(f[q z]-f[z]) \\
& +\frac{(a-z)(b-z)(c-z)(d-z)}{\left(1-z^{2}\right)\left(q-z^{2}\right)}\left(f\left[q^{-1} z\right]-f[z]\right)+\left(1+q^{-1} a b c d\right) f[z] .
\end{aligned}
$$

The eigenvalue equation is

$$
D_{\mathrm{sym}} P_{n}=\lambda_{n} P_{n}, \quad \lambda_{n}:=q^{-n}+a b c d q^{n-1} .
$$

Under condition (1.1) all eigenvalues in (2.3) are distinct.

The three-term recurrence relation for the monic Askey-Wilson polynomials is

$$
\left(z+z^{-1}\right) P_{n}[z]=P_{n+1}[z]+\beta_{n} P_{n}[z]+\gamma_{n} P_{n-1}[z] \quad(n=0,1,2, \ldots),
$$

where $\beta_{n}$ and $\gamma_{n}$ are suitable constants and $P_{-1}[z]:=0$ (see $[6,(3.1 .5)]$ ).

\subsection{Zhedanov's algebra}

Zhedanov's algebra $A W(3)$ (see $[15,5]$ ) can in the $q$-case be described as an algebra with two generators $K_{0}, K_{1}$ and with two relations

$$
\begin{aligned}
& \left(q+q^{-1}\right) K_{1} K_{0} K_{1}-K_{1}^{2} K_{0}-K_{0} K_{1}^{2}=B K_{1}+C_{0} K_{0}+D_{0}, \\
& \left(q+q^{-1}\right) K_{0} K_{1} K_{0}-K_{0}^{2} K_{1}-K_{1} K_{0}^{2}=B K_{0}+C_{1} K_{1}+D_{1} .
\end{aligned}
$$

Here the structure constants $B, C_{0}, C_{1}, D_{0}, D_{1}$ are fixed complex constants.

There is a Casimir operator $Q$ commuting with $K_{0}, K_{1}$ :

$$
\begin{aligned}
Q= & K_{1} K_{0} K_{1} K_{0}-\left(q^{2}+1+q^{-2}\right) K_{0} K_{1} K_{0} K_{1}+\left(q+q^{-1}\right) K_{0}^{2} K_{1}^{2} \\
& +\left(q+q^{-1}\right)\left(C_{0} K_{0}^{2}+C_{1} K_{1}^{2}\right)+B\left(\left(q+1+q^{-1}\right) K_{0} K_{1}+K_{1} K_{0}\right) \\
& +\left(q+1+q^{-1}\right)\left(D_{0} K_{0}+D_{1} K_{1}\right) .
\end{aligned}
$$


Let the structure constants be expressed in terms of $a, b, c, d$ by means of $e_{1}, e_{2}, e_{3}, e_{4}$ (see (1.2)) as follows:

$$
\begin{aligned}
& B:=\left(1-q^{-1}\right)^{2}\left(e_{3}+q e_{1}\right), \\
& C_{0}:=\left(q-q^{-1}\right)^{2}, \\
& C_{1}:=q^{-1}\left(q-q^{-1}\right)^{2} e_{4}, \\
& D_{0}:=-q^{-3}(1-q)^{2}(1+q)\left(e_{4}+q e_{2}+q^{2}\right), \\
& D_{1}:=-q^{-3}(1-q)^{2}(1+q)\left(e_{1} e_{4}+q e_{3}\right) .
\end{aligned}
$$

Then there is a representation (the basic representation or polynomial representation) of the algebra $A W(3)$ with structure constants (2.7) on the space $\mathcal{A}_{\text {sym }}$ of symmetric Laurent polynomials as follows:

$$
\left(K_{0} f\right)[z]:=\left(D_{\mathrm{sym}} f\right)[z], \quad\left(K_{1} f\right)[z]:=\left(Z+Z^{-1}\right) f[z]:=\left(z+z^{-1}\right) f[z],
$$

where $D_{\text {sym }}$ is the operator (2.2) having the Askey-Wilson polynomials as eigenfunctions. The Casimir operator $Q$ becomes constant in this representation:

$$
(Q f)(z)=Q_{0} f(z)
$$

where

$$
\begin{aligned}
Q_{0}:= & q^{-4}(1-q)^{2}\left(q^{4}\left(e_{4}-e_{2}\right)+q^{3}\left(e_{1}^{2}-e_{1} e_{3}-2 e_{2}\right)\right. \\
& \left.-q^{2}\left(e_{2} e_{4}+2 e_{4}+e_{2}\right)+q\left(e_{3}^{2}-2 e_{2} e_{4}-e_{1} e_{3}\right)+e_{4}\left(1-e_{2}\right)\right) .
\end{aligned}
$$

(Note the slight error in this formula in [7, (2.8)], version v3. It is corrected in v4.)

Remark 2.1. Write $A W(3)=A W\left(3 ; K_{0}, K_{1} ; a, b, c, d ; q\right)$ in order to emphasize the dependence of $A W(3)$ on the generators and the parameters (by (2.4), (2.5), (2.7)). There are several symmetries of this algebra. First of all it is invariant under permutations of $a, b, c, d$. The following one will be compatible with the duality of the double affine Hecke algebra to be discussed below:

There is an anti-algebra isomorphism

$$
\begin{aligned}
A W\left(3 ; K_{0}, K_{1} ; a, b, c, d ; q\right) \rightarrow & A W\left(3 ; a K_{1},\left(q^{-1} a b c d\right)^{-\frac{1}{2}} K_{0} ;\right. \\
& \left.\frac{1}{\left(q^{-1} a b c d\right)^{\frac{1}{2}}}, \frac{a b}{\left(q^{-1} a b c d\right)^{\frac{1}{2}}}, \frac{a c}{\left(q^{-1} a b c d\right)^{\frac{1}{2}}}, \frac{a d}{\left(q^{-1} a b c d\right)^{\frac{1}{2}}} ; q\right) .
\end{aligned}
$$

See $[15, \S 2]$. Under this mapping $Q$ is sent to $q a(b c d)^{-1} Q$ and $Q_{0}$ to $q a(b c d)^{-1} Q_{0}$.

Note that there is also a trivial anti-algebra isomorphism

$$
A W\left(3 ; K_{0}, K_{1} ; a, b, c, d ; q\right) \rightarrow A W\left(3 ; K_{0}, K_{1} ; a, b, c, d ; q\right) .
$$

This sends $Q$ to $Q$ and $Q_{0}$ to $Q_{0}$.

There is also an algebra isomorphism

$$
A W\left(3 ; K_{0}, K_{1} ; a, b, c, d ; q\right) \rightarrow A W\left(3 ; \frac{q}{a b c d} K_{0}, K_{1} ; a^{-1}, b^{-1}, c^{-1}, d^{-1} ; q^{-1}\right) .
$$

This sends $Q$ to $q^{2}(a b c d)^{-2} Q$ and $Q_{0}$ to $q^{2}(a b c d)^{-2} Q_{0}$. 
Let $A W\left(3, Q_{0}\right)$ be the algebra generated by $K_{0}, K_{1}$ with relations $(2.4),(2.5)$ and $Q=Q_{0}$, assuming the structure constants (2.7). Then the basic representation of $A W(3)$ is also a representation of $A W\left(3, Q_{0}\right)$. We have the following theorem (see [7, Theorem 2.2]):

Theorem 2.2. The elements

$$
K_{0}^{n}\left(K_{1} K_{0}\right)^{l} K_{1}^{m} \quad(m, n=0,1,2, \ldots, l=0,1)
$$

form a basis of $A W\left(3, Q_{0}\right)$ and the representation (2.8) of $A W\left(3, Q_{0}\right)$ is faithful.

Note that the anti-algebra isomorphism (2.11) induces an anti-algebra isomorphisms for $A W\left(3, Q_{0}\right)$.

\subsection{The double affine Hecke algebra of type $\left(C_{1}^{\vee}, C_{1}\right)$}

One of the ways to describe the double affine Hecke algebra of type $\left(C_{1}^{\vee}, C_{1}\right)$, denoted by $\tilde{\mathfrak{H}}$, is as follows (see [7, Proposition 5.2]):

Definition 2.3. $\tilde{\mathfrak{H}}$ is the algebra generated by $T_{1}, Y, Y^{-1}, Z, Z^{-1}$ with relations $Y Y^{-1}=1=$ $Y^{-1} Y, Z Z^{-1}=1=Z^{-1} Z$ and

$$
\begin{aligned}
& T_{1}^{2}=-(a b+1) T_{1}-a b, \\
& T_{1} Z=Z^{-1} T_{1}+(a b+1) Z^{-1}-(a+b), \\
& T_{1} Z^{-1}=Z T_{1}-(a b+1) Z^{-1}+(a+b), \\
& T_{1} Y=q^{-1} a b c d Y^{-1} T_{1}-(a b+1) Y+a b\left(1+q^{-1} c d\right), \\
& T_{1} Y^{-1}=q(a b c d)^{-1} Y T_{1}+q(a b c d)^{-1}(1+a b) Y-q(c d)^{-1}\left(1+q^{-1} c d\right), \\
& Y Z=q Z Y+(1+a b) c d Z^{-1} Y^{-1} T_{1}-(a+b) c d Y^{-1} T_{1}-\left(1+q^{-1} c d\right) Z^{-1} T_{1} \\
& \quad-(1-q)(1+a b)\left(1+q^{-1} c d\right) Z^{-1}+(c+d) T_{1}+(1-q)(a+b)\left(1+q^{-1} c d\right), \\
& Y Z^{-1}=q^{-1} Z^{-1} Y-q^{-2}(1+a b) c d Z^{-1} Y^{-1} T_{1}+q^{-2}(a+b) c d Y^{-1} T_{1} \\
& \quad+q^{-1}\left(1+q^{-1} c d\right) Z^{-1} T_{1}-q^{-1}(c+d) T_{1}, \\
& \quad+q(a b c d)^{-1}\left(1+q^{-1} c d\right) Z^{-1} T_{1}+q(a b c d)^{-1}(1-q)(1+a b)\left(1+q^{-1} c d\right) Z^{-1} \\
& \quad-(a b c d)^{-1}(c+d) T_{1}-(a b c d)^{-1}(1-q)(1+a b)(c+d), \\
& \quad-q^{-1} Z Y^{-1}-q(a b)^{-1}\left(1+a b Z^{-1} Y^{-1} T_{1}+(a b)^{-1}(a+b) Y^{-1} T_{1}\right. \\
& \quad q^{-1}(a b c d)^{-1}\left(1+q^{-1} c d\right) Z^{-1} T_{1}+q(a b c d)^{-1}(c+d) T_{1} .
\end{aligned}
$$

By adding the relations for $T Z$ and $T Z^{-1}$ and by combining the relations for $T Y$ and $T Y^{-1}$ we see that

$$
T_{1}\left(Z+Z^{-1}\right)=\left(Z+Z^{-1}\right) T_{1}, \quad T_{1}\left(Y+q^{-1} a b c d Y^{-1}\right)=\left(Y+q^{-1} a b c d Y^{-1}\right) T_{1} .
$$

For the following theorem see Sahi [12] in the general rank case. In [7, Theorem 5.3] it is proved for the rank one case only.

Theorem 2.4. A basis of $\tilde{\mathfrak{H}}$ is provided by the elements $Z^{m} Y^{n} T_{1}^{i}$, where $m, n \in \mathbb{Z}, i=0,1$.

The basic or polynomial representation of $\tilde{\mathfrak{H}}$ is a representation of $\tilde{\mathfrak{H}}$ on the space $\mathcal{A}$ of Laurent polynomials $f[z]$ in $z$ such that $(Z f)[z]:=z f[z]$ and $T_{1}$ and $Y$ act as $q$-difference-reflection operators given by $[7,(3.11),(3.13)]$. This representation is faithful. If $a b \neq 1$ then $T_{1}$ acting 
on $\mathcal{A}$ has eigenspaces $\mathcal{A}_{\text {sym }}$ (for eigenvalue $\left.-a b\right)$ and $\mathcal{A}_{\text {sym }}^{-}$(for eigenvalue -1 ), and $\mathcal{A}$ is the direct sum of $\mathcal{A}_{\text {sym }}$ and $\mathcal{A}_{\text {sym }}^{-}$.

In the basic representation of $\tilde{\mathfrak{H}}$ the operator $Y+q^{-1} a b c d Y^{-1}$ has eigenvalues $\lambda_{n}$ (see (2.3)), of multiplicity 2 for $n=1,2, \ldots$ and of multiplicity 1 for $n=0$. If $n \geq 1$ and $a b \neq 1$ then the eigenspace of $\lambda_{n}$ splits as a one-dimensional part in $\mathcal{A}_{\text {sym }}$ spanned by the Askey-Wilson polynomial $P_{n}[z]$ given by $(2.1)$ and a one-dimensional part in $\mathcal{A}_{\text {sym }}^{-}$spanned by

$$
Q_{n}[z]:=a^{-1} b^{-1} z^{-1}(1-a z)(1-b z) P_{n-1}[z ; q a, q b, c, d \mid q] .
$$

In $[7, \S 6]$ the following algebra $\widetilde{A W}\left(3, Q_{0}\right)$ was defined, which is a central extension of $A W\left(3, Q_{0}\right)$ :

Definition 2.5. The algebra $\widetilde{A W}\left(3, Q_{0}\right)$ is generated by $K_{0}, K_{1}, T_{1}$ such that $\left(T_{1}+a b\right)\left(T_{1}+1\right)=$ $0, T_{1}$ commutes with $K_{0}, K_{1}$, and with further relations

$$
\begin{aligned}
(q+ & \left.q^{-1}\right) K_{1} K_{0} K_{1}-K_{1}^{2} K_{0}-K_{0} K_{1}^{2}=B K_{1}+C_{0} K_{0}+D_{0} \\
& +E K_{1}\left(T_{1}+a b\right)+F_{0}\left(T_{1}+a b\right), \\
(q+ & \left.q^{-1}\right) K_{0} K_{1} K_{0}-K_{0}^{2} K_{1}-K_{1} K_{0}^{2}=B K_{0}+C_{1} K_{1}+D_{1} \\
& +E K_{0}\left(T_{1}+a b\right)+F_{1}\left(T_{1}+a b\right), \\
Q_{0}= & \left(K_{1} K_{0}\right)^{2}-\left(q^{2}+1+q^{-2}\right) K_{0}\left(K_{1} K_{0}\right) K_{1}+\left(q+q^{-1}\right) K_{0}^{2} K_{1}^{2} \\
& +\left(q+q^{-1}\right)\left(C_{0} K_{0}^{2}+C_{1} K_{1}^{2}\right)+\left(B+E\left(T_{1}+a b\right)\right)\left(\left(q+1+q^{-1}\right) K_{0} K_{1}+K_{1} K_{0}\right) \\
& +\left(q+1+q^{-1}\right)\left(D_{0}+F_{0}\left(T_{1}+a b\right)\right) K_{0}+\left(q+1+q^{-1}\right)\left(D_{1}+F_{1}\left(T_{1}+a b\right)\right) K_{1} \\
& +G\left(T_{1}+a b\right) .
\end{aligned}
$$

Here the structure constants are given by (2.7) together with

$$
\begin{aligned}
E:= & -q^{-2}(1-q)^{3}(c+d), \\
F_{0}:= & q^{-3}(1-q)^{3}(1+q)(c d+q), \\
F_{1}:= & q^{-3}(1-q)^{3}(1+q)(a+b) c d, \\
G:= & -q^{-4}(1-q)^{3}\left((a+b)(c+d)\left(c d\left(q^{2}+1\right)+q\right)-q(a b+1)\left(\left(c^{2}+d^{2}\right)(q+1)-c d\right)\right. \\
& \left.+\left(c d+e_{4}\right)\left(q^{2}+1\right)+\left(e_{2}+e_{4}-a b\right) q^{3}\right),
\end{aligned}
$$

and $Q_{0}$ is given by $(2.10)$.

Then it was proved in [7, Theorem 6.2, Corollary 6.3]:

Theorem 2.6. $\widetilde{A W}\left(3, Q_{0}\right)$ has a basis consisting of

$$
K_{0}^{n}\left(K_{1} K_{0}\right)^{i} K_{1}^{m} T_{1}^{j} \quad(m, n=0,1,2, \ldots, i, j=0,1) .
$$

There is a unique algebra isomorphism from $\widetilde{A W}\left(3, Q_{0}\right)$ into $\tilde{\mathfrak{H}}$ such that $K_{0} \mapsto Y+q^{-1} a b c d Y^{-1}$, $K_{1} \mapsto Z+Z^{-1}, T_{1} \mapsto T_{1}$. The elements in the image commute with $T_{1}$.

Remark 2.7. Write $\tilde{\mathfrak{H}}=\tilde{\mathfrak{H}}\left(Y, Z, T_{1} ; a, b, c, d ; q\right)$ in order to emphasize the dependence of $\tilde{\mathfrak{H}}$ on the generators and the parameters. Then there is an anti-algebra isomorphism

$$
\begin{aligned}
\tilde{\mathfrak{H}}\left(Y, Z, T_{1} ; a, b, c, d ; q\right) \rightarrow & \tilde{\mathfrak{H}}\left(a Z^{-1},\left(q^{-1} a b c d\right)^{-\frac{1}{2}} Y^{-1}, T_{1} ;\right. \\
& \left.\frac{1}{\left(q^{-1} a b c d\right)^{\frac{1}{2}}}, \frac{a b}{\left(q^{-1} a b c d\right)^{\frac{1}{2}}}, \frac{a c}{\left(q^{-1} a b c d\right)^{\frac{1}{2}}}, \frac{a d}{\left(q^{-1} a b c d\right)^{\frac{1}{2}}} ; q\right),
\end{aligned}
$$


see $[9$, Proposition $8.5(i)]$. Also write $\widetilde{A W}\left(3, Q_{0}\right)=\widetilde{A W}\left(3, Q_{0} ; K_{0}, K_{1}, T_{1} ; a, b, c, d ; q\right)$. Then, by a slight adaptation of (2.11), there is an anti-algebra isomorphism

$$
\begin{aligned}
\widetilde{A W}\left(3, Q_{0} ; K_{0}, K_{1}, T_{1} ; a, b, c, d ; q\right) \rightarrow & \widetilde{A W}\left(3 ; q a(b c d)^{-1} Q_{0} ; a K_{1},\left(q^{-1} a b c d\right)^{-\frac{1}{2}} K_{0} ;\right. \\
& \left.\frac{1}{\left(q^{-1} a b c d\right)^{\frac{1}{2}}}, \frac{a b}{\left(q^{-1} a b c d\right)^{\frac{1}{2}}}, \frac{a c}{\left(q^{-1} a b c d\right)^{\frac{1}{2}}}, \frac{a d}{\left(q^{-1} a b c d\right)^{\frac{1}{2}}} ; q\right) .
\end{aligned}
$$

The two anti-algebra isomorphisms are compatible under the algebra embedding of $\widetilde{A W}\left(3, Q_{0}\right)$ into $\tilde{\mathfrak{H}}$ given in Theorem 2.6 .

\section{The spherical subalgebra}

From now on assume $a b \neq 1$. In $\tilde{\mathfrak{H}}$ put

$$
P_{\text {sym }}:=(1-a b)^{-1}\left(T_{1}+1\right) .
$$

Then

$$
P_{\mathrm{sym}}^{2}=P_{\mathrm{sym}} .
$$

In the basic representation of $\tilde{\mathfrak{H}}$ we have for $f \in \mathcal{A}$ :

$$
P_{\mathrm{sym}} f= \begin{cases}f & \text { if } T_{1} f=-a b f \\ 0 & \text { if } T_{1} f=-f .\end{cases}
$$

$P_{\text {sym }}$ projects $\mathcal{A}$ onto $\mathcal{A}_{\text {sym }}$. Define the linear map $S: \tilde{\mathfrak{H}} \rightarrow \tilde{\mathfrak{H}}$ by

$$
S(U):=P_{\mathrm{sym}} U P_{\mathrm{sym}} \quad(U \in \tilde{\mathfrak{H}}) .
$$

Then

$$
S(U) S(V)=S\left(U P_{\mathrm{sym}} V\right) \quad(U, V \in \tilde{\mathfrak{H}}) .
$$

Hence the image $S(\tilde{\mathfrak{H}})$ is a subalgebra of $\tilde{\mathfrak{H}}$. We call it the spherical subalgebra of $\tilde{\mathfrak{H}}$.

For $U \in \tilde{\mathfrak{H}}$ we have in the basic representation:

$$
S(U) f=P_{\mathrm{sym}} U P_{\mathrm{sym}} f= \begin{cases}P_{\mathrm{sym}} U f & \text { if } T_{1} f=-a b f \\ 0 & \text { if } T_{1} f=-f .\end{cases}
$$

Hence, for the basic representation of $\tilde{\mathfrak{H}}$ restricted to $S(\tilde{\mathfrak{H}}), \mathcal{A}_{\text {sym }}$ is an invariant subspace. This representation of $S(\tilde{\mathfrak{H}})$ on $\mathcal{A}_{\text {sym }}$ is faithful. Indeed, if $S(U) f=0$ for all $f \in \mathcal{A}_{\text {sym }}$ then $S(U) f=0$ for all $f \in \mathcal{A}$, so $S(U)=0$ by the faithfulness of the basic representation of $\tilde{\mathfrak{H}}$ on $\mathcal{A}$. $Z_{\tilde{\mathfrak{H}}}\left(T_{1}\right)$, the centralizer of $T_{1}$ in $\tilde{\mathfrak{H}}$, is a subalgebra of $\tilde{\mathfrak{H}}$. It has $P_{\text {sym }}$ as a central element. Hence

$$
S(U)=U P_{\mathrm{sym}} \quad \text { and } \quad S(U V)=S(U) S(V) \quad\left(U, V \in Z_{\tilde{\mathfrak{H}}}\left(T_{1}\right)\right) .
$$

So $S$ restricted to $Z_{\tilde{\mathfrak{H}}}\left(T_{1}\right)$ is an algebra homomorphism.

The algebra $\widetilde{A W}\left(3, Q_{0}\right)$ was defined by Definition 2.5. By Theorem 2.6 there is an algebra isomorphism from $\widehat{A W}\left(3, Q_{0}\right)$ into $\tilde{\mathfrak{H}}$ such that $K_{0} \mapsto Y+q^{-1} a b c d Y^{-1}, K_{1} \mapsto Z+Z^{-1}$, 
$T_{1} \mapsto T_{1}$. This isomorphism embeds $\widetilde{A W}\left(3, Q_{0}\right)$ into $Z_{\tilde{\mathfrak{H}}}\left(T_{1}\right)$. So we may consider $\widetilde{A W}\left(3, Q_{0}\right)$ as a subalgebra of $Z_{\tilde{\mathfrak{H}}}\left(T_{1}\right)$ and (3.1) will hold for $U, V \in \widehat{A W}\left(3, Q_{0}\right)$.

By (2.4), (2.5), (2.9) and (2.6), the algebra $A W\left(3, Q_{0}\right)$ can be presented by the same generators and relations as for $\widetilde{A W}\left(3, Q_{0}\right)$ but with additional relation $T_{1}=-a b$.

By Theorem 2.6 $\widetilde{A W}\left(3, Q_{0}\right)$ has a basis consisting of the elements

$$
K_{0}^{n}\left(K_{1} K_{0}\right)^{i} K_{1}^{m}\left(T_{1}+1\right), \quad K_{0}^{n}\left(K_{1} K_{0}\right)^{i} K_{1}^{m}\left(T_{1}+a b\right) \quad(m, n=0,1,2, \ldots, \quad i=0,1) .
$$

Hence $S\left(\widetilde{A W}\left(3, Q_{0}\right)\right)$ has a basis consisting of

$$
(1-a b)^{-1} K_{0}^{n}\left(K_{1} K_{0}\right)^{i} K_{1}^{m}\left(T_{1}+1\right) \quad(m, n=0,1,2, \ldots, \quad i=0,1) .
$$

By Theorem $2.2 A W\left(3, Q_{0}\right)$ has a basis consisting of

$$
K_{0}^{n}\left(K_{1} K_{0}\right)^{i} K_{1}^{m} \quad(m, n=0,1,2, \ldots, \quad i=0,1) .
$$

Hence the map which sends a basis element $K_{0}^{n}\left(K_{1} K_{0}\right)^{i} K_{1}^{m}$ of $A W\left(3, Q_{0}\right)$ to a basis element $(1-a b)^{-1} K_{0}^{n}\left(K_{1} K_{0}\right)^{i} K_{1}^{m}\left(T_{1}+1\right)$ of $S\left(\widetilde{A W}\left(3, Q_{0}\right)\right)$ extends linearly to a linear bijection from $A W\left(3, Q_{0}\right)$ onto $S\left(\widetilde{A W}\left(3, Q_{0}\right)\right)$. In fact, this map remains well-defined if we write it as

$$
U \mapsto(1-a b)^{-1} \widetilde{U}\left(T_{1}+1\right),
$$

where $U \mapsto \widetilde{U}$ sends words $U$ in $A W\left(3, Q_{0}\right)$ to corresponding words $\widetilde{U}$ in $\widetilde{A W}\left(3, Q_{0}\right)$. Moreover, the map (3.3) can then be seen to be an algebra homomorphism. Indeed, consider the linear map $U \mapsto \widetilde{U}$ as a map to $\widetilde{A W}\left(3, Q_{0}\right)$ from the free algebra generated by $K_{0}, K_{1}, T_{1}$ with $T_{1}$ central such that it sends a word involving $K_{0}, K_{1}, T_{1}$ to the same word in $\widetilde{A W}\left(3, Q_{0}\right)$. This map is an algebra homomorphism. Composing it with $S$ yields the map (3.3) which is again an algebra homomorphism. Now we have to check that $R$ is sent to zero by the map (3.3) if $R=0$ is a relation for $A W\left(3, Q_{0}\right)$. This is clearly the case for $R:=T_{1}+a b$, since $\left(T_{1}+a b\right)\left(T_{1}+1\right)=0$. It is also clear for the other relations $R=0$ in $A W\left(3, Q_{0}\right)$ since these can be taken as the relations (2.14)-(2.16), which are also relations for $\widetilde{A W}\left(3, Q_{0}\right)$. So we have shown:

Proposition 3.1. The map (3.3), where $U \mapsto \widetilde{U}$ sends words $U$ involving $K_{0}, K_{1}$ in $A W\left(3, Q_{0}\right)$ to the same words $\widetilde{U}$ in $\widetilde{A W}\left(3, Q_{0}\right)$, is a well defined algebra isomorphism from $A W\left(3, Q_{0}\right)$ onto $S\left(\widetilde{A W}\left(3, Q_{0}\right)\right)$.

Theorem 3.2. $S(\tilde{\mathfrak{H}})=S\left(\widetilde{A W}\left(3, Q_{0}\right)\right)$, so the spherical subalgebra $S(\tilde{\mathfrak{H}})$ is isomorphic to the algebra $A W\left(3, Q_{0}\right)$ by the map (3.3) sending $A W\left(3, Q_{0}\right)$ to $S(\tilde{\mathfrak{H}})$.

For the proof note first that $\tilde{\mathfrak{H}}$ has a basis consisting of the elements

$$
Z^{m} Y^{n}\left(T_{1}+1\right), \quad Z^{m} Y^{n}\left(T_{1}+a b\right) \quad(m, n \in \mathbb{Z}) .
$$

Hence $S(\tilde{\mathfrak{H}})$ is spanned by the elements

$$
\left(T_{1}+1\right) Z^{m} Y^{n}\left(T_{1}+1\right) \quad(m, n \in \mathbb{Z}) .
$$

Definition 3.3. Let $m, n \in \mathbb{Z}$. For an element in $\tilde{\mathfrak{H}}$ which is a linear combination of basis elements $Z^{k} Y^{l}$ we say that

$$
\sum_{k, l \in \mathbb{Z}} c_{k, l} Z^{k} Y^{l}=o\left(Z^{m} Y^{n}\right)
$$

if $c_{k, l} \neq 0$ implies $|k| \leq|m|,|l| \leq|n|,(|k|,|l|) \neq(|m|,|n|)$. 
Theorem 3.2 will follow by induction with respect to $|m|+|n|$ from the following lemma:

Lemma 3.4. Let $m, n \in \mathbb{Z}$. Then

$$
\left(T_{1}+1\right) Z^{m} Y^{n}\left(T_{1}+1\right) \in\left(T_{1}+1\right)\left(\widetilde{A W}\left(3, Q_{0}\right)+o\left(Z^{m} Y^{n}\right)\right)\left(T_{1}+1\right) .
$$

Proof. The procedure will be as follows:

1. Write $\left(T_{1}+1\right) Z^{m} Y^{n}\left(T_{1}+1\right)$ as a linear combination of

$$
\begin{array}{lc}
Z^{|m|} Y^{|n|}\left(T_{1}+1\right), & Z^{-|m|} Y^{|n|}\left(T_{1}+1\right), \\
Z^{-|m|} Y^{-|n|}\left(T_{1}+1\right) & \left(\bmod o\left(Z^{|m|} Y^{|n|}\right)\left(T_{1}+1\right)\right) .
\end{array}
$$

This is done by induction, starting with the $\tilde{\mathfrak{H}}$ relations for $T_{1} Z, T_{1} Z^{-1}, T_{1} Y, T_{1} Y^{-1}$.

2. Also write $K_{1}^{m} K_{0}^{n}\left(T_{1}+1\right)$ and $K_{1}^{m-1} K_{0} K_{1} K_{0}^{n-1}\left(T_{1}+1\right)(m, n=0,1, \ldots)$ as a linear combination of (3.5).

3. These latter linear combinations turn out to span the linear combinations obtained for $\left(T_{1}+1\right) Z^{m} Y^{n}\left(T_{1}+1\right)$.

Step 1. We get the following expressions in terms of the elements (3.5) (here $m, n \in\{1,2, \ldots\}$ )

$$
\begin{aligned}
\left(T_{1}+1\right) Z^{m}\left(T_{1}+1\right)=\left(Z^{m}+Z^{-m}+o\left(Z^{m}\right)\right)\left(T_{1}+1\right) & \\
\left(T_{1}+1\right) Z^{-m}\left(T_{1}+1\right)=-a b\left(Z^{m}+Z^{-m}+o\left(Z^{m}\right)\right)\left(T_{1}+1\right) & \\
\left(T_{1}+1\right) Y^{n}\left(T_{1}+1\right)=-a b\left(Y^{n}+\left(q^{-1} a b c d\right)^{n} Y^{-n}+o\left(Y^{n}\right)\right)\left(T_{1}+1\right) & \left(\left(q^{-1} a b c d\right)^{-n} Y^{n}+Y^{-n}+o\left(Y^{n}\right)\right)\left(T_{1}+1\right), \\
\left(T_{1}+1\right) Y^{-n}\left(T_{1}+1\right)= & \left(Z^{m} Y^{n}-a b\left(q^{-1} a b c d\right)^{n} Z^{-m} Y^{-n}+o\left(Z^{m} Y^{n}\right)\right)\left(T_{1}+1\right), \\
\left(T_{1}+1\right) Z^{m} Y^{n}\left(T_{1}+1\right) & \left(-(a b+1) Z^{m} Y^{n}-a b\left(q^{-1} a b c d\right)^{n} Z^{m} Y^{-n}-a b Z^{-m} Y^{n}\right. \\
\left(T_{1}+1\right) Z^{-m} Y^{n}\left(T_{1}+1\right)= & \left.\left(Z^{m} Y^{n}\right)\right)\left(T_{1}+1\right), \\
\left(T_{1}+1\right) Z^{m} Y^{-n}\left(T_{1}+1\right)= & \left(Z^{m} Y^{-n}+\left(q^{-1} a b c d\right)^{-n} Z^{-m} Y^{n}+(1+a b) Z^{-m} Y^{-n}\right. \\
& \left.+o\left(Z^{m} Y^{n}\right)\right)\left(T_{1}+1\right), \\
\left(T_{1}+1\right) Z^{-m} Y^{-n}\left(T_{1}+1\right)= & \left(\left(q^{-1} a b c d\right)^{-n} Z^{m} Y^{n}-a b Z^{-m} Y^{-n}\right. \\
& \left.+o\left(Z^{m} Y^{n}\right)\right)\left(T_{1}+1\right) .
\end{aligned}
$$

Step 2. We get the following expressions in terms of the elements $(3.5)$ (here $m, n \in\{1,2, \ldots\}$ )

$$
\begin{aligned}
& K_{1}^{m}\left(T_{1}+1\right)=\left(Z^{m}+Z^{-m}+o\left(Z^{m}\right)\right)\left(T_{1}+1\right), \\
& K_{0}^{n}\left(T_{1}+1\right)=\left(Y^{n}+\left(q^{-1} a b c d\right)^{n} Y^{-n}+o\left(Y^{n}\right)\right)\left(T_{1}+1\right) \\
& K_{1}^{m} K_{0}^{n}\left(T_{1}+1\right)=\left(Z^{m} Y^{n}+Z^{-m} Y^{n}+\left(q^{-1} a b c d\right)^{n}\left(Z^{m} Y^{-n}+Z^{-m} Y^{-n}\right)\right. \\
& \left.\quad+o\left(Z^{m} Y^{n}\right)\right)\left(T_{1}+1\right), \\
& \left.\quad+q^{-1}\left(q^{-1} a b c d\right)^{n}\left(1+a b-q^{2} a b\right) Z^{-m} Y^{-n}+o\left(Z^{m} Y^{n}\right)\right)\left(T_{1}+1\right) .
\end{aligned}
$$


Step 3. The only cases which may not be immediately clear are for $\left(T_{1}+1\right) Z^{ \pm m} Y^{ \pm n}\left(T_{1}+1\right)$ $(m, n \in\{1,2, \ldots\})$. Then we have by comparing the identities in Step 1 and Step 2:

$$
\begin{aligned}
\left(T_{1}+1\right) Z^{m} Y^{n}\left(T_{1}+1\right)= & \frac{1}{\left(1-q^{2}\right)}\left(K_{1}^{m-1}\left(K_{1} K_{0}-q K_{0} K_{1}\right) K_{0}^{n-1}+o\left(Z^{m} Y^{n}\right)\right)\left(T_{1}+1\right), \\
\left(T_{1}+1\right) Z^{-m} Y^{n}\left(T_{1}+1\right)= & \frac{q}{\left(1-q^{2}\right)}\left(K_{1}^{m-1}\left(-q^{-1}\left(1+a b-q^{2} a b\right) K_{1} K_{0}+K_{0} K_{1}\right) K_{0}^{n-1}\right. \\
& \left.+o\left(Z^{m} Y^{n}\right)\right)\left(T_{1}+1\right), \\
\left(T_{1}+1\right) Z^{m} Y^{-n}\left(T_{1}+1\right)= & \frac{q}{\left(1-q^{2}\right)\left(q^{-1} a b c d\right)^{n}}\left(K_{1}^{m-1}\left(-q K_{1} K_{0}+K_{0} K_{1}\right) K_{0}^{n-1}\right. \\
& \left.+o\left(Z^{m} Y^{n}\right)\right)\left(T_{1}+1\right), \\
\left(T_{1}+1\right) Z^{-m} Y^{-n}\left(T_{1}+1\right)= & \frac{1}{\left(1-q^{2}\right)\left(q^{-1} a b c d\right)^{n}}\left(K_{1}^{m-1}\left(K_{1} K_{0}-q K_{0} K_{1}\right) K_{0}^{n-1}\right. \\
& \left.+o\left(Z^{m} Y^{n}\right)\right)\left(T_{1}+1\right) .
\end{aligned}
$$

Proofs for Step 1. We will use repeatedly that $\left(T_{1}+1\right)^{2}=(1-a b)\left(T_{1}+1\right)$. From the relation for $T_{1} Z$ in (2.12) we obtain

$$
\begin{aligned}
& \left(T_{1}+1\right) Z=Z^{-1}\left(T_{1}+1\right)+Z+a b Z^{-1}-(a+b), \\
& \left(T_{1}+1\right) Z\left(T_{1}+1\right)=\left(Z+Z^{-1}-(a+b)\right)\left(T_{1}+1\right)=\left(Z+Z^{-1}+o(Z)\right)\left(T_{1}+1\right),
\end{aligned}
$$

i.e., (3.6) for $m=1$. Now we will prove (3.6) by induction. Suppose it holds for some positive integer $m$, then we will prove the identity with $m$ replaced by $m+1$. By successively substituting (3.18) and the induction hypothesis we have:

$$
\begin{aligned}
\left(T_{1}+1\right) Z^{m+1}\left(T_{1}+1\right) & =Z^{-1}\left(T_{1}+1\right) Z^{m}\left(T_{1}+1\right)+\left(Z^{m+1}+o\left(Z^{m+1}\right)\right)\left(T_{1}+1\right) \\
& =Z^{-1}\left(Z^{m}+Z^{-m}+o\left(Z^{m}\right)\right)\left(T_{1}+1\right)+\left(Z^{m+1}+o\left(Z^{m+1}\right)\right)\left(T_{1}+1\right) \\
& =\left(Z^{m+1}+Z^{-m-1}+o\left(Z^{m+1}\right)\right)\left(T_{1}+1\right) .
\end{aligned}
$$

Similarly, from the relation for $T_{1} Z^{-1}$ in $(2.12)$ we prove (3.7) by induction:

$$
\begin{aligned}
& \left(T_{1}+1\right) Z^{-1}=Z\left(T_{1}+1\right)-Z-a b Z^{-1}+(a+b), \quad \text { hence } \\
& \left(T_{1}+1\right) Z^{-1}\left(T_{1}+1\right)=\left(-a b\left(Z+Z^{-1}\right)+(a+b)\right)\left(T_{1}+1\right) \\
& =-a b\left(Z+Z^{-1}+o(Z)\right)\left(T_{1}+1\right), \\
& \left(T_{1}+1\right) Z^{-m-1}\left(T_{1}+1\right)=Z\left(T_{1}+1\right) Z^{-m}\left(T_{1}+1\right)-a b\left(Z^{-m-1}+o\left(Z^{m+1}\right)\right)\left(T_{1}+1\right) \\
& =-a b Z\left(Z^{m}+Z^{-m}+o\left(Z^{m}\right)\right)\left(T_{1}+1\right)-a b\left(Z^{-m-1}+o\left(Z^{m+1}\right)\right)\left(T_{1}+1\right) \\
& =-a b\left(Z^{m+1}+Z^{-m-1}+o\left(Z^{m+1}\right)\right)\left(T_{1}+1\right) .
\end{aligned}
$$

The proofs of (3.8), (3.9) are similar.

The proof of (3.10) is for fixed $n$ by induction with respect to $m$. First we prove the case $m=1$. By (3.18) and (3.8) we obtain:

$$
\begin{aligned}
\left(T_{1}+1\right) Z Y^{n}\left(T_{1}+1\right) & =\left(Z^{-1}\left(T_{1}+1\right) Y^{n}+Z Y^{n}+a b Z^{-1} Y^{n}+o\left(Z Y^{n}\right)\right)\left(T_{1}+1\right) \\
& =\left(Z Y^{n}-a b\left(q^{-1} a b c d\right)^{n} Z^{-1} Y^{-n}+o\left(Z Y^{n}\right)\right)\left(T_{1}+1\right) .
\end{aligned}
$$

Now suppose (3.10) holds for some positive integer $m$, then we will prove the identity with $m$ replaced by $m+1$. By (3.18) and the induction hypothesis we obtain:

$$
\begin{aligned}
\left(T_{1}+1\right) Z^{m+1} Y^{n}\left(T_{1}+1\right) & =\left(Z^{-1}\left(T_{1}+1\right) Z^{m} Y^{n}+Z^{m+1} Y^{n}+o\left(Z^{m+1} Y^{n}\right)\right)\left(T_{1}+1\right) \\
& =\left(Z^{m+1} Y^{n}-a b\left(q^{-1} a b c d\right)^{n} Z^{-m-1} Y^{-n}+o\left(Z^{m+1} Y^{n}\right)\right)\left(T_{1}+1\right) .
\end{aligned}
$$


The proofs of (3.11), (3.12), (3.13) are similar.

Proofs for Step 2. Formulas (3.14), (3.15), (3.16) immediately follow by the substitutions $K_{0}=Y+q^{-1} a b c d Y^{-1}, K_{1}=Z+Z^{-1}$. As for (3.17) we first verify it for $m=n=1$ by writing the left-hand side as $(1-a b)^{-1}\left(T_{1}+1\right)\left(Y+q^{-1} a b c d Y^{-1}\right)\left(Z+Z^{-1}\right)\left(T_{1}+1\right)$ and by using the last four relations in (2.12). Then we obtain for the case of general $m, n$ :

$$
\begin{aligned}
& K_{1}^{m-1} K_{0} K_{1} K_{0}^{n-1}\left(T_{1}+1\right)=(1-a b)^{-1} K_{1}^{m-1} K_{0} K_{1}\left(T_{1}+1\right) K_{0}^{n-1}\left(T_{1}+1\right) \\
&=\left(Z+Z^{-1}\right)^{m-1}\left(q Z Y+q^{-1} Z^{-1} Y+q^{-1}\left(q^{-1} a b c d\right) Z Y^{-1}\right. \\
&\left.\quad+q^{-1}\left(q^{-1} a b c d\right)\left(1+a b-q^{2} a b\right) Z^{-1} Y^{-1}+o(Z Y)\right)\left(Y+q^{-1} a b c d Y^{-1}\right)^{n-1}\left(T_{1}+1\right) \\
&=\left(q Z^{m} Y^{n}+q^{-1} Z^{-m} Y^{n}+q^{-1}\left(q^{-1} a b c d\right)^{n} Z^{m} Y^{-n}\right. \\
&\left.\quad+q^{-1}\left(q^{-1} a b c d\right)^{n}\left(1+a b-q^{2} a b\right) Z^{-m} Y^{-n}+o\left(Z^{m} Y^{n}\right)\right)\left(T_{1}+1\right) .
\end{aligned}
$$

\section{The antispherical subalgebra}

In $\tilde{\mathfrak{H}}$ put

$$
P_{\text {sym }}^{-}:=(a b-1)^{-1}\left(T_{1}+a b\right) .
$$

Then

$$
\left(P_{\mathrm{sym}}^{-}\right)^{2}=P_{\mathrm{sym}}^{-}
$$

In the basic representation of $\tilde{\mathfrak{H}}$ we have for $f \in \mathcal{A}$ :

$$
P_{\text {sym }}^{-} f= \begin{cases}f & \text { if } T_{1} f=-f, \\ 0 & \text { if } T_{1} f=-a b f .\end{cases}
$$

Let $\mathcal{A}_{\text {sym }}^{-}$denote the eigenspace of $T_{1}$ acting on $\mathcal{A}$ for eigenvalue -1 . Then $P_{\text {sym }}^{-}$projects $\mathcal{A}$ onto $\mathcal{A}_{\text {sym }}^{-}$. Define the linear map $S^{-}: \tilde{\mathfrak{H}} \rightarrow \tilde{\mathfrak{H}}$ by

$$
S^{-}(U):=P_{\text {sym }}^{-} U P_{\text {sym }}^{-} \quad(U \in \tilde{\mathfrak{H}}) .
$$

Then

$$
S^{-}(U) S^{-}(V)=S^{-}\left(U P_{\text {sym }}^{-} V\right) \quad(U, V \in \tilde{\mathfrak{H}}) .
$$

Hence the image $S^{-}(\tilde{\mathfrak{H}})$ is a subalgebra of $\tilde{\mathfrak{H}}$. We call it the antispherical subalgebra of $\tilde{\mathfrak{H}}$.

For $U \in \tilde{\mathfrak{H}}$ we have in the basic representation:

$$
S^{-}(U) f=P_{\mathrm{sym}}^{-} U P_{\mathrm{sym}}^{-} f= \begin{cases}P_{\mathrm{sym}}^{-} U f & \text { if } T_{1} f=-f, \\ 0 & \text { if } T_{1} f=-a b f .\end{cases}
$$

Hence, for the basic representation of $\tilde{\mathfrak{H}}$ restricted to $S^{-}(\tilde{\mathfrak{H}}), \mathcal{A}_{\text {sym }}^{-}$is an invariant subspace.

Recall the algebra isomorphism from $\widetilde{A W}\left(3, Q_{0}\right)$ into $Z_{\tilde{\mathfrak{H}}}\left(T_{1}\right)$ given by Theorem 2.6. Since $S^{-}(U) S^{-}(V)=S^{-}(U V)$ for $U, V \in Z_{\tilde{\mathfrak{H}}}\left(T_{1}\right)$, we see that $\widehat{A W}\left(3, Q_{0}\right)$, considered as a subalgabra of $\tilde{\mathfrak{H}}$ and hence of $Z_{\tilde{\mathfrak{H}}}\left(T_{1}\right)$, is mapped by $S^{-}$onto a subalgebra $S^{-}\left(\widetilde{A W}\left(3, Q_{0}\right)\right)$ of $S^{-}(\tilde{\mathfrak{H}})$. From the basis $(3.2)$ of $\widetilde{A W}\left(3, Q_{0}\right)$ we see that $S^{-}\left(\widetilde{A W}\left(3, Q_{0}\right)\right)$ has basis

$$
(a b-1)^{-1} K_{0}^{n}\left(K_{1} K_{0}\right)^{i} K_{1}^{m}\left(T_{1}+a b\right) \quad(m, n=0,1,2, \ldots, i=0,1) .
$$


Compare this basis with the basis (3.2) of $A W\left(3, Q_{0}\right)$. Thus the map which sends a basis element $K_{0}^{n}\left(K_{1} K_{0}\right)^{i} K_{1}^{m}$ of $A W\left(3, Q_{0}\right)$ to a basis element $q^{n+i}(a b-1)^{-1} K_{0}^{n}\left(K_{1} K_{0}\right)^{i} K_{1}^{m}\left(T_{1}+a b\right)$ of $S^{-}\left(\widetilde{A W}\left(3, Q_{0}\right)\right)$ extends linearly to a linear bijection from $A W\left(3, Q_{0}\right)$ onto $S^{-}\left(\widetilde{A W}\left(3, Q_{0}\right)\right)$. In fact this map extends to an algebra isomorphism:

Proposition 4.1. Let $A W\left(3, Q_{0} ; q a, q b, c, d\right)$ be $A W\left(3, Q_{0}\right)$ with $a, b$ replaced by $q a$, $q b$, respectively. Then there is a well-defined algebra isomorphism from $A W\left(3, Q_{0} ; q a, q b, c, d\right)$ onto $S^{-}\left(\widetilde{A W}\left(3, Q_{0}\right)\right)$ given by the map

$$
U \mapsto(a b-1)^{-1} \widetilde{U}\left(T_{1}+a b\right),
$$

where $U \mapsto \widetilde{U}$ sends words $U$ in $A W\left(3, Q_{0} ; q a, q b, c, d\right)$ involving $K_{0}, K_{1}$ to corresponding words $\widetilde{U}$ in $\widetilde{A W}\left(3, Q_{0}\right)$ involving $q K_{0}, K_{1}$.

Proof. Consider the linear map $U \mapsto \widetilde{U}$ as a map to $\widetilde{A W}\left(3, Q_{0}\right)$ from the free algebra generated by $K_{0}, K_{1}, T_{1}$ with $T_{1}$ central such that it sends a word involving $K_{0}, K_{1}, T_{1}$ to the corresponding word involving $q K_{0}, K_{1}, T_{1}$ in $\widetilde{A W}\left(3, Q_{0}\right)$. This map is an algebra homomorphism. Composing it with $S^{-}$yields the map (4.1) which is again an algebra homomorphism. Now we have to check that $R$ is sent to zero by the map (4.1) if $R=0$ is a relation for $A W\left(3, Q_{0} ; q a, q b, c, d\right)$. This is clearly the case for $R:=T_{1}+1$, since $\left(T_{1}+1\right)\left(T_{1}+a b\right)=0$. To see this for the other relations, rewrite relations $(2.14)-(2.16)$ for $\widetilde{A W}\left(3, Q_{0}\right)$ as:

$$
\begin{aligned}
& \left(q+q^{-1}\right) K_{1} K_{0} K_{1}-K_{1}^{2} K_{0}-K_{0} K_{1}^{2}-(B+(a b-1) E) K_{1}-C_{0} K_{0}-\left(D_{0}+(a b-1) F_{0}\right) \\
& \quad-\left(E K_{1}+F_{0}\right)\left(T_{1}+1\right)=0, \\
& \quad\left(q+q^{-1}\right) K_{0} K_{1} K_{0}-K_{0}^{2} K_{1}-K_{1} K_{0}^{2}-(B+(a b-1) E) K_{0}-C_{1} K_{1}-\left(D_{1}+(a b-1) F_{1}\right) \\
& \quad-\left(E K_{0}+F_{1}\right)\left(T_{1}+1\right)=0, \\
& \quad K_{1} K_{0} K_{1} K_{0}-\left(q^{2}+1+q^{-2}\right) K_{0} K_{1} K_{0} K_{1}+\left(q+q^{-1}\right) K_{0}^{2} K_{1}^{2}-\left(\left(q+q^{-1}\right)\left(C_{0} K_{0}^{2}+C_{1} K_{1}^{2}\right)\right. \\
& \quad+(B+(a b-1) E)\left(\left(q+1+q^{-1}\right) K_{0} K_{1}+K_{1} K_{0}\right)+\left(q+1+q^{-1}\right)\left(D_{0}+(a b-1) F_{0}\right) K_{0} \\
& \quad+\left(q+1+q^{-1}\right)\left(D_{1}+(a b-1) F_{1}\right) K_{1}+(a b-1) G-Q_{0}+\left(E K_{1} K_{0}+G\right)\left(T_{1}+1\right) \\
& \quad+\left(q+1+q^{-1}\right)\left(E K_{0} K_{1}+F_{0} K_{0}+F_{1} K_{1}\right)\left(T_{1}+1\right)=0 .
\end{aligned}
$$

On multiplication with $T_{1}+a b$ we see that the identities $R_{i}\left(T_{1}+a b\right)=0(i=1,2,3)$ must be valid in $S^{-}\left(\widetilde{A W}\left(3, Q_{0}\right)\right)$, where

$$
\begin{aligned}
R_{1}:= & \left(q+q^{-1}\right) K_{1} K_{0} K_{1}-K_{1}^{2} K_{0}-K_{0} K_{1}^{2}-(B+(a b-1) E) K_{1} \\
& -C_{0} K_{0}-D_{0}-(a b-1) F_{0}, \\
R_{2}:= & \left(q+q^{-1}\right) K_{0} K_{1} K_{0}-K_{0}^{2} K_{1}-K_{1} K_{0}^{2}-(B+(a b-1) E) K_{0} \\
& -C_{1} K_{1}-D_{1}-(a b-1) F_{1}, \\
R_{3}:= & K_{1} K_{0} K_{1} K_{0}-\left(q^{2}+1+q^{-2}\right) K_{0} K_{1} K_{0} K_{1}+\left(q+q^{-1}\right) K_{0}^{2} K_{1}^{2} \\
& -\left(\left(q+q^{-1}\right)\left(C_{0} K_{0}^{2}+C_{1} K_{1}^{2}\right)+(B+(a b-1) E)\left(\left(q+1+q^{-1}\right) K_{0} K_{1}+K_{1} K_{0}\right)\right. \\
& +\left(q+1+q^{-1}\right)\left(D_{0}+(a b-1) F_{0}\right) K_{0}+\left(q+1+q^{-1}\right)\left(D_{1}+(a b-1) F_{1}\right) K_{1} \\
& +(a b-1) G-Q_{0} .
\end{aligned}
$$

Now consider relations (2.4), (2.5) en (2.9) for $A W\left(3, Q_{0} ; q a, q b, c, d\right)$ (so with $a, b$ in the structure constants replaced by $q a, q b)$. These can be written in the form $U_{i}=0(i=1,2,3)$ by bringing everything to the left-hand side in the relations. Now consider $U_{i}$ as elements of the free algebra generated by $K_{0}, K_{1}$. For the images under the map (4.1) we then obtain the following elements of $S^{-}\left(\widetilde{A W}\left(3, Q_{0}\right)\right)$ :

$$
\widetilde{U}_{1}=(1-a b)^{-1} q R_{1}, \quad \widetilde{U}_{2}=(1-a b)^{-1} q^{2} R_{2}, \quad \widetilde{U}_{3}=(1-a b)^{-1} q^{2} R_{3},
$$

which are all zero. 
Theorem 4.2. $S^{-}(\tilde{\mathfrak{H}})=S\left(\widetilde{A W}\left(3, Q_{0}\right)\right)$, so the antispherical subalgebra $S^{-}(\tilde{\mathfrak{H}})$ is isomorphic to the algebra $A W\left(3, Q_{0} ; q a, q b, c, d\right)$ by the map (4.1) sending $A W\left(3, Q_{0} ; q a, q b, c, d\right)$ to $S^{-}(\tilde{\mathfrak{H}})$.

The proof is analogous to the proof of Theorem 3.2. Since $\tilde{\mathfrak{H}}$ has a basis $(3.4), S^{-}(\tilde{\mathfrak{H}})$ is spanned by the elements

$$
\left(T_{1}+a b\right) Z^{m} Y^{n}\left(T_{1}+a b\right) \quad(m, n \in \mathbb{Z}) .
$$

Recall Definition 3.3. Theorem 4.2 will follow by induction with respect to $|m|+|n|$ from the following analogue of Lemma 3.4:

Lemma 4.3. Let $m, n \in \mathbb{Z}$. Then

$$
\left(T_{1}+a b\right) Z^{m} Y^{n}\left(T_{1}+a b\right) \in\left(T_{1}+a b\right)\left(\widetilde{A W}\left(3, Q_{0}\right)+o\left(Z^{m} Y^{n}\right)\right)\left(T_{1}+a b\right) .
$$

Proof. We use the same procedure as in the proof of Lemma 3.4. I will only list the main formulas in the three steps. The reader can verify these formulas in an analogous way as in the proof of Lemma 3.4 .

\section{Step 1}

$$
\begin{aligned}
\left(T_{1}+a b\right) Z^{m}\left(T_{1}+a b\right)=a b( & \left.Z^{m}+Z^{-m}+o\left(Z^{m}\right)\right)\left(T_{1}+a b\right) \\
\left(T_{1}+a b\right) Z^{-m}\left(T_{1}+a b\right)=- & \left(Z^{m}+Z^{-m}+o\left(Z^{m}\right)\right)\left(T_{1}+a b\right), \\
\left(T_{1}+a b\right) Y^{n}\left(T_{1}+a b\right)=-\left(Y^{n}+\left(q^{-1} a b c d\right)^{n} Y^{-n}+o\left(Y^{n}\right)\right)\left(T_{1}+a b\right), & \\
\left(T_{1}+a b\right) Y^{-n}\left(T_{1}+a b\right)=a b( & \left.\left(q^{-1} a b c d\right)^{-n} Y^{n}+Y^{-n}+o\left(Y^{n}\right)\right)\left(T_{1}+a b\right), \\
\left(T_{1}+a b\right) Z^{m} Y^{n}\left(T_{1}+a b\right)= & \left(a b Z^{m} Y^{n}-\left(q^{-1} a b c d\right)^{n} Z^{-m} Y^{-n}+o\left(Z^{m} Y^{n}\right)\right)\left(T_{1}+a b\right), \\
\left(T_{1}+a b\right) Z^{-m} Y^{n}\left(T_{1}+a b\right)= & \left(-(a b+1) Z^{m} Y^{n}-\left(q^{-1} a b c d\right)^{n} Z^{m} Y^{-n}-Z^{-m} Y^{n}\right. \\
& \left.+o\left(Z^{m} Y^{n}\right)\right)\left(T_{1}+a b\right), \\
\left(T_{1}+a b\right) Z^{m} Y^{-n}\left(T_{1}+a b\right)= & \left(a b\left(q^{-1} a b c d\right)^{-n} Z^{-m} Y^{n}+a b Z^{m} Y^{-n}+(1+a b) Z^{-m} Y^{-n}\right. \\
& \left.+o\left(Z^{m} Y^{n}\right)\right)\left(T_{1}+a b\right), \\
\left(T_{1}+a b\right) Z^{-m} Y^{-n}\left(T_{1}+a b\right)= & \left(a b\left(q^{-1} a b c d\right)^{-n} Z^{m} Y^{n}-Z^{-m} Y^{-n}+o\left(Z^{m} Y^{n}\right)\right)\left(T_{1}+a b\right) .
\end{aligned}
$$

\section{Step 2}

$$
\begin{aligned}
& K_{1}^{m}\left(T_{1}+a b\right)=\left(Z^{m}+Z^{-m}+o\left(Z^{m}\right)\right)\left(T_{1}+a b\right), \\
& K_{0}^{n}\left(T_{1}+a b\right)=\left(Y^{n}+\left(q^{-1} a b c d\right)^{n} Y^{-n}+o\left(Y^{n}\right)\right)\left(T_{1}+a b\right), \\
& K_{1}^{m} K_{0}^{n}\left(T_{1}+a b\right)=\left(Z^{m} Y^{n}+Z^{-m} Y^{n}+\left(q^{-1} a b c d\right)^{n}\left(Z^{m} Y^{-n}+Z^{-m} Y^{-n}\right)\right. \\
& \left.\quad+o\left(Z^{m} Y^{n}\right)\right)\left(T_{1}+a b\right), \\
& \left.\quad+(q a b)^{-1}\left(q^{-1} a b c d\right)^{n}\left(1+a b-q^{2}\right) Z^{-m} Y^{-n}+o\left(Z^{m} Y^{n}\right)\right)\left(T_{1}+a b\right) .
\end{aligned}
$$




\section{Step 3}

$$
\begin{aligned}
\left(T_{1}+a b\right) Z^{m} Y^{n}\left(T_{1}+a b\right)= & \frac{a b}{1-q^{2}}\left(K_{1}^{m-1}\left(K_{1} K_{0}-q K_{0} K_{1}\right) K_{0}^{n-1}+o\left(Z^{m} Y^{n}\right)\right)\left(T_{1}+a b\right), \\
\left(T_{1}+a b\right) Z^{-m} Y^{n}\left(T_{1}+a b\right)= & \frac{1}{1-q^{2}}\left(K_{1}^{m-1}\left(-\left(1+a b-q^{2}\right) K_{1} K_{0}+q a b K_{0} K_{1}\right) K_{0}^{n-1}\right. \\
& \left.+o\left(Z^{m} Y^{n}\right)\right)\left(T_{1}+a b\right), \\
\left(T_{1}+a b\right) Z^{m} Y^{-n}\left(T_{1}+a b\right)= & \frac{q a b}{\left(1-q^{2}\right)\left(q^{-1} a b c d\right)^{n}}\left(K_{1}^{m-1}\left(-q K_{1} K_{0}+K_{0} K_{1}\right) K_{0}^{n-1}\right. \\
& \left.+o\left(Z^{m} Y^{n}\right)\right)\left(T_{1}+a b\right), \\
\left(T_{1}+a b\right) Z^{-m} Y^{-n}\left(T_{1}+a b\right)= & \frac{a b}{\left(1-q^{2}\right)\left(q^{-1} a b c d\right)^{n}}\left(K_{1}^{m-1}\left(K_{1} K_{0}-q K_{0} K_{1}\right) K_{0}^{n-1}\right. \\
& \left.+o\left(Z^{m} Y^{n}\right)\right)\left(T_{1}+a b\right) .
\end{aligned}
$$

Remark 4.4. The same $q$-shift for the parameters as in Proposition 4.1 occurs in $[7,(3.17)]$ (the eigenfunction of $Y+q^{-1} a b c d Y^{-1}$ in $\mathcal{A}_{\text {sym }}^{-}$expressed in terms of Askey-Wilson polynomials). Of course, these two results are very much related to each other.

Let $\tilde{\mathfrak{H}}(q a, q b, c, d)$ be $\tilde{\mathfrak{H}}$ with parameters $a, b, c, d$ replaced by $q a, q b, c, d$, respectively. If we compare Theorems 3.2 and 4.2 then we can conclude that the spherical subalgebras $S^{-}(\tilde{\mathfrak{H}})$ and $S(\tilde{\mathfrak{H}}(q a, q b, c, d))$ are isomorphic. This result is an analogue of the result in [1, Proposition 4.11] for Cherednik algebras.

Remark 4.5. Just as we had in Step 1 of the proofs of Lemma 3.4 and Lemma 4.3, we can derive from the first, fourth and fifth relation in (2.12) that

$$
\begin{aligned}
& \left(T_{1}+1\right)\left(Y+q^{-1} a^{2} b^{2} c d Y^{-1}-\left(q^{-1} a b c d+a b\right)\right)\left(T_{1}+1\right)=0 \\
& \left(T_{1}+a b\right)\left(Y+q^{-1} c d Y^{-1}-\left(q^{-1} c d+1\right)\right)\left(T_{1}+a b\right)=0 .
\end{aligned}
$$

It follows that, in the basic representation of $\tilde{\mathfrak{H}}$, the operator

$$
D^{-}:=Y+q^{-1} a^{2} b^{2} c d Y^{-1}-\left(q^{-1} a b c d+a b\right)
$$

maps $\mathcal{A}_{\text {sym }}$ into $\mathcal{A}_{\text {sym }}^{-}$, while

$$
D^{+}:=Y+q^{-1} c d Y^{-1}-\left(q^{-1} c d+1\right)
$$

maps $\mathcal{A}_{\text {sym }}^{-}$into $\mathcal{A}_{\text {sym }}$. Since both operators preserve the eigenspace of $\lambda_{n}$, we obtain

$$
\begin{aligned}
& D^{-} P_{n}=a b\left(q^{-1} c d-q^{-n}\right)\left(q^{n}-1\right) Q_{n}, \\
& D^{+} Q_{n}=\left(q^{-1} c d-q^{-n} a^{-1} b^{-1}\right)\left(q^{n} a b-1\right) P_{n},
\end{aligned}
$$

where the Askey-Wilson polynomial $P_{n}$ and the shifted Askey-Wilson polynomial $Q_{n}$ are given by (2.1) and (2.13), respectively, and the constant factors in the above identities follow by comparing coefficients of $z^{n}$. Thus the operators $D^{-}$and $D^{+}$can be considered as shift operators.

The observations in this Remark were earlier made (in different notation) in [9, Lemma 12.2, Proposition 12.3]. 


\section{Centralizers and centers}

As a corollary of Theorem 3.2 and Theorem 4.2 we obtain:

Theorem 5.1. The centralizer $Z_{\tilde{\mathfrak{H}}}\left(T_{1}\right)$ is equal to $\widetilde{A W}\left(3, Q_{0}\right)$.

Proof. Write $U \in \tilde{\mathfrak{H}}$ as

$$
U=(1-a b)^{-1} U\left(T_{1}+1\right)+(a b-1)^{-1} U\left(T_{1}+a b\right) .
$$

Suppose that $U \in Z_{\tilde{\mathfrak{H}}}\left(T_{1}\right)$. Then

$$
\begin{aligned}
& U\left(T_{1}+1\right)=(1-a b)^{-1}\left(T_{1}+1\right) U\left(T_{1}+1\right), \\
& U\left(T_{1}+a b\right)=(a b-1)^{-1}\left(T_{1}+a b\right) U\left(T_{1}+a b\right) .
\end{aligned}
$$

So $U\left(T_{1}+1\right) \in S(\tilde{\mathfrak{H}})=S\left(\widetilde{A W}\left(3, Q_{0}\right)\right) \subset \widetilde{A W}\left(3, Q_{0}\right)$ and $U\left(T_{1}+a b\right) \in S^{-}(\tilde{\mathfrak{H}})=S^{-}\left(\widetilde{A W}\left(3, Q_{0}\right)\right) \subset$ $\widetilde{A W}\left(3, Q_{0}\right)$.

The following theorem is interesting in its own right, but it can also be used, in combination with Theorems 3.2 and 4.2, in order to show that the center of $\tilde{\mathfrak{H}}$ consists of the scalars (see Theorem 5.3). The proof is in the same spirit as the proof of the faithfulness of the basic representation of $A W\left(3, Q_{0}\right)$ (see [7, Theorem 2.2]).

Theorem 5.2. The center of the algebra $A W\left(3, Q_{0}\right)$ consists of the scalars.

Proof. Let $U$ be in the center of $A W\left(3, Q_{0}\right)$. Because of Theorem 2.2 we may consider $A W\left(3, Q_{0}\right)$ in its faithful basic representation on $\mathcal{A}_{\text {sym }}$. Then $U$ can be uniquely expanded in terms of the basis of $A W\left(3, Q_{0}\right)$ in this representation:

$$
U=\sum_{k, l} a_{k, l} D_{\mathrm{sym}}^{l}\left(Z+Z^{-1}\right)^{k}+\sum_{k, l} b_{k, l} D_{\mathrm{sym}}^{l-1}\left(Z+Z^{-1}\right) D_{\mathrm{sym}}\left(Z+Z^{-1}\right)^{k-1} .
$$

Since $U$ is in the center, we have

$$
U\left(Z+Z^{-1}\right)-\left(Z+Z^{-1}\right) U=0 .
$$

We will first show that if $U$ given by (5.2) satisfies (5.3), then all coefficients $a_{k, l}$ and $b_{k, l}$ in (5.2) vanish except possibly for coefficients $a_{k, l}$ with $l=0$. Indeed, suppose that this is not the case. Then there is a highest value $m$ of $k$ for which $a_{k, l} \neq 0$ or $b_{k, l} \neq 0$ for some $l \geq 1$. All terms in (5.2) with $k>m$ then will certainly commute with $Z+Z^{-1}$, so we may assume that the terms in (5.2) with $k>m$ vanish while (5.3) still holds. Let both sides of (5.3) act on the Askey-Wilson polynomial $P_{j}[z]$ :

$$
\left(U\left(Z+Z^{-1}\right)-\left(Z+Z^{-1}\right) U\right) P_{j}[z]=0 .
$$

Expand the left-hand side of the above equation in terms of Askey-Wilson polynomials $P_{i}[z]$. Then the highest occurring term will be for $i=j+m+1$, so the coefficient of $P_{j+m+1}[z]$ in this expansion must be zero. This gives

$$
\sum_{l}\left(a_{m, l} \lambda_{j+m+1}^{l}+b_{m, l} \lambda_{j+m+1}^{l-1} \lambda_{j+m}\right)-\sum_{l}\left(a_{m, l} \lambda_{j+m}^{l}+b_{m, l} \lambda_{j+m}^{l-1} \lambda_{j+m-1}\right)=0 .
$$

We have, writing $x:=q^{j+m}$ and $u:=q^{-1} a b c d$,

$$
\lambda_{j+m+1}=q^{-1} x^{-1}+q u x, \quad \lambda_{j+m}=x^{-1}+u x, \quad \lambda_{j+m-1}=q x^{-1}+q^{-1} u x .
$$


We can consider the identity (5.4) as an identity for Laurent polynomials in $x$. Since the lefthand side vanishes for infinitely many values of $x$, it must be identically zero. Let $n$ be the maximal $l>0$ for which $a_{m, l} \neq 0$ or $b_{m, l} \neq 0$. Then, in particular, the coefficients of $x^{-n}$ and $x^{n}$ in the left-hand side of (5.4) must be zero. This gives explicitly:

$$
a_{m, n} u^{n}\left(1-q^{n}\right)+q^{-1} b_{m, n} u^{n}\left(1-q^{n}\right)=0, \quad a_{m, n}\left(1-q^{-n}\right)+q b_{m, n}\left(1-q^{-n}\right)=0 .
$$

Now $n>0$, so $q^{ \pm n} \neq 1$. Also $u \neq 0$. Hence,

$$
a_{m, n}+q^{-1} b_{m, n}=0, \quad a_{m, n}+q b_{m, n}=0 .
$$

Thus $a_{m, n}=0=b_{m, n}$, which is a contradiction.

So $U$ in the center will have the form $U=\sum_{k} a_{k}\left(Z+Z^{-1}\right)^{k}$ in the basic representation of $A W\left(3, Q_{0}\right)$. We have to show that $a_{k}=0$ for $k>0$. Suppose not. Then there is a highest value $m>0$ of $k$ for which $a_{k} \neq 0$. Then we have

$$
U D_{\text {sym }}(1)-D_{\text {sym }} U(1)=0 \text {. }
$$

Expand the left-hand side of the above equation in terms of Askey-Wilson polynomials $P_{i}[z]$. Then the coefficient of $P_{m}[z]$ will be zero. So $a_{m} \lambda_{0}-a_{m} \lambda_{m}=0$. Since $\lambda_{m} \neq \lambda_{0}$ if $m \neq 0$, we conclude that $a_{m}=0$, a contradiction.

Theorem 5.3. The center $Z(\tilde{\mathfrak{H}})$ of $\tilde{\mathfrak{H}}$ consists of the scalars.

Proof. Let $U \in Z(\tilde{\mathfrak{H}})$. Then $U \in Z_{\tilde{\mathfrak{H}}}\left(T_{1}\right)=\widetilde{A W}\left(3, Q_{0}\right)$. So $U \in Z\left(\widetilde{A W}\left(3, Q_{0}\right)\right)$. Write $U$ as in (5.1). We have to show that $U\left(T_{1}+1\right)$ and $U\left(T_{1}+a b\right)$ are scalars. This follows from

$$
\begin{aligned}
& U\left(T_{1}+1\right)=(1-a b)^{-1} S(U) \in Z(S(\tilde{\mathfrak{H}})), \\
& U\left(T_{1}+a b\right)=(a b-1)^{-1} S^{-}(U) \in Z\left(S^{-}(\tilde{\mathfrak{H}})\right) .
\end{aligned}
$$

Now use the algebra isomorphisms from Theorems 3.2 and 4.2, and apply Theorem 5.2.

\section{Acknowledgements}

I thank Jasper Stokman for suggesting me that the spherical subalgebra of the Askey-Wilson DAHA is related to Zhedanov's algebra. I thank a referee for suggestions which led to inclusion of Remarks 2.1, 2.7 and 4.5. Some of the results presented here were obtained during the workshop Applications of Macdonald Polynomials, September 9-14, 2007 at the Banff International Research Station (BIRS). I thank the organizers for inviting me.

\section{References}

[1] Berest Y., Etingof P., Ginzburg V., Cherednik algebras and differential operators on quasi-invariants, Duke Math. J. 118 (2003), 279-337, math.QA/0111005.

[2] Cherednik I., Double affine Hecke algebras, Knizhnik-Zamolodchikov equations, and Macdonald's operators, Int. Math. Res. Not. 1992 (1992), no. 9, 171-180.

[3] Etingof P., Ginzburg V., Symplectic reflection algebras, Calogero-Moser space, and deformed HarishChandra homomorphism, Invent. Math. 147 (2002), 243-348, math.AG/0011114.

[4] Gasper G., Rahman M., Basic hypergeometric series, 2nd ed., Cambridge University Press, 2004.

[5] Granovskiı̌ Ya.I., Lutzenko I.M., Zhedanov A.S., Mutual integrability, quadratic algebras, and dynamical symmetry, Ann. Physics 217 (1992), 1-20.

[6] Koekoek R., Swarttouw R.F., The Askey-scheme of hypergeometric orthogonal polynomials and its $q$ analogue, Report 98-17, Faculty of Technical Mathematics and Informatics, Delft University of Technology, 1998, http://aw.twi.tudelft.nl/ koekoek/askey/. 
[7] Koornwinder T.H., The relationship between Zhedanov's algebra $A W(3)$ and the double affine Hecke algebra in the rank one case, SIGMA 3 (2007), 063, 15 pages, math.QA/0612730.

[8] Macdonald I.G., Affine Hecke algebra and orthogonal polynomials, Cambridge University Press, 2003.

[9] Noumi M., Stokman J.V., Askey-Wilson polynomials: an affine Hecke algebraic approach, in Laredo Lectures on Orthogonal Polynomials and Special Functions, Nova Sci. Publ., Hauppauge, NY, 2004, 111-144, math.QA/0001033.

[10] Oblomkov A., Double affine Hecke algebras of rank 1 and affine cubic surfaces, Int. Math. Res. Not. 2004 (2004), 877-912, math.RT/0306393.

[11] Opdam E.M., Some applications of hypergeometric shift operators, Invent. Math. 98 (1989), 1-18.

[12] Sahi S., Nonsymmetric Koornwinder polynomials and duality, Ann. of Math. (2) 150 (1999), 267-282, q-alg/9710032.

[13] Sahi S., Some properties of Koornwinder polynomials, in $q$-Series from a Contemporary Perspective, Contemp. Math. 254 (2000), 395-411.

[14] Sahi S., Raising and lowering operators for Askey-Wilson polynomials, SIGMA 3 (2007), 002, 11 pages, math.QA/0701134.

[15] Zhedanov A.S., "Hidden symmetry" of Askey-Wilson polynomials, Theoret. and Math. Phys. 89 (1991), 1146-1157. 